\title{
Aiding the diagnosis of dissociative identity disorder: pattern recognition study of brain biomarkers
}

Antje A. T. S. Reinders*, Andre F. Marquand*, Yolanda R. Schlumpft, Sima Chalavit, Eline M. Vissia, Ellert R. S. Nijenhuis, Paola Dazzan, Lutz Jäncke and Dick J. Veltman

\section{Background}

A diagnosis of dissociative identity disorder (DID) is controversial and prone to under- and misdiagnosis. From the moment of seeking treatment for symptoms to the time of an accurate diagnosis of DID individuals received an average of four prior other diagnoses and spent 7 years, with reports of up to 12 years, in mental health services.

\section{Aim}

To investigate whether data-driven pattern recognition methodologies applied to structural brain images can provide biomarkers to aid DID diagnosis.

\section{Method}

Structural brain images of 75 participants were included: 32 female individuals with DID and 43 matched healthy controls. Individuals with DID were recruited from psychiatry and psychotherapy out-patient clinics. Probabilistic pattern classifiers were trained to discriminate cohorts based on measures of brain morphology.

\section{Results}

The pattern classifiers were able to accurately discriminate between individuals with DID and healthy controls with high sensitivity (72\%) and specificity (74\%) on the basis of brain structure. These findings provide evidence for a biological basis for distinguishing between DID-affected and healthy individuals.

\section{Conclusions}

We propose a pattern of neuroimaging biomarkers that could be used to inform the identification of individuals with DID from healthy controls at the individual level. This is important and clinically relevant because the DID diagnosis is controversial and individuals with DID are often misdiagnosed. Ultimately, the application of pattern recognition methodologies could prevent unnecessary suffering of individuals with DID because of an earlier accurate diagnosis, which will facilitate faster and targeted interventions.

\section{Declaration of interest}

The authors declare no competing financial interests.

\section{Keywords}

Magnetic resonance imaging (MRI); childhood trauma; post-traumatic stress disorder (PTSD); dissociation.

\section{Copyright and usage}

(c) The Royal College of Psychiatrists 2018
Dissociative identity disorder (DID) ${ }^{1}$ is probably the most disputed psychiatric disorder. ${ }^{2,3}$ DID is the most severe of the dissociative disorders and involves two or more dissociative personality states as well as recurrent gaps in the recall of everyday events, important personal information and/or traumatic events that are inconsistent with ordinary forgetting and is not related to substance misuse or general medication. For decades the disorder has been officially recognised in the DSM (in the DSM-III (1980) as Multiple Personality Disorder), but many patients with DID share a history of years of misdiagnoses, inefficient pharmacological treatment and several admissions to hospital. ${ }^{4}$ To date, many clinicians and scientists still question the validity and even the very existence of DID. ${ }^{5,6}$ Unfamiliarity with the spectrum of dissociative disorders, lack of knowledge and appreciation of its epidemiology, ${ }^{7}$ the existence of imitative DID as well as the reluctance of individuals with DID to present their dissociative symptoms, often due to feelings of shame, may lead to the validity concerns and under- and misdiagnosis of DID. Years of incorrect treatment results in protracted personal suffering and high direct and indirect societal costs. ${ }^{8,9}$ Structural brain imaging holds promise to aid disease diagnosis by providing objective biomarkers at the single-subject level, ${ }^{10}$ thereby paving the way for a fast and correct diagnosis of individuals with DID. The current study presents a first step towards an automated classification of individuals with DID by investigating

* These authors contributed equally to this article.

† These authors contributed equally to this article. whether individuals with DID can be separated from healthy controls on the basis of neuroimaging markers, thus informing DID's neuroanatomical basis in terms of grey and white matter of the brain.

Sceptics of DID assume that dissociative symptoms can easily be simulated by normal healthy individuals, which comprises the Fantasy Model of DID. ${ }^{11}$ Several pioneering functional brain imaging studies from our group have shown that individuals with genuine DID can be distinguished from healthy controls simulating DID, ${ }^{12-14}$ but critics could still argue that people can manipulate their brain activity. ${ }^{15,16}$ As neuroanatomical data is unlikely to be subject to cognitive manipulation, in this study we investigate whether people with DID can be separated from healthy controls at the individual level on the basis of neuroanatomical biomarkers by employing a multivariate data-driven pattern recognition approach. We used a probabilistic pattern recognition approach ${ }^{10}$ that allowed us to investigate the diagnostic value of grey and white matter of the brain in a comparatively large sample of individuals with DID as compared with healthy controls, and to quantify the degree to which the brain phenotype of people with DID can be distinguished from those of healthy people. This study is important in two ways: (a) it provides evidence as to whether genuine DID can be distinguished from normal healthy individuals on the basis of their brain morphology, thereby addressing the core of the Fantasy Model for DID; and (b) it provides neuroanatomical biomarkers that could support the development of pattern recognition methodologies to be used as a clinically useful aid for the diagnostic accuracy of DID. 


\section{Method}

\section{Participants}

Magnetic resonance imaging (MRI) data from 75 participants were obtained in the Netherlands in the University Medical Centre in Groningen (UMCG) and the Amsterdam Medical Centre (AMC), and in Switzerland at the University Hospital in Zurich. Details of these two samples have previously been published elsewhere: Chalavi et $a l^{17,18}$ (Dutch sample), Schlumpf et al ${ }^{12,13}$ (Swiss/ German sample) and Reinders et al ${ }^{19}$ (combined). All participants gave informed written consent in accordance with the Declaration of Helsinki and as dictated by ethics approval which was obtained from the Medical Ethical Committees of UMCG (Reference number: METC2008.211) and the AMC (Reference number: MEC09/155), and by the cantonal ethical commission of Zurich (Kantonale Ethikkommission Zürich; reference number: E-13/2008).

Overall, 32 women with DID were recruited from private practitioners of psychiatry and psychotherapy and psychiatric outpatient departments. They were initially diagnosed according to DSM-IV criteria for DID. ${ }^{20}$ The clinical diagnosis was subsequently confirmed by independent expert clinicians using the Structural Clinical Interview for DSM-IV Dissociative Disorders (SCID-D), ${ }^{21,22}$ specifically to avoid the inclusion of imitative DID. ${ }^{23}$ DID is known to be highly comorbid. ${ }^{24,25}$ In the current study posttraumatic stress disorder (PTSD) comorbidity was debriefed using the SCID-D and it was found that out of the 32 DID individuals, 29 had comorbid PTSD and 3 had PTSD in remission. The following information concerning other comorbid disorders was obtained based on DSM-IV (1994) classification from the participants and/or their personal therapists $(N=29)$ : no other comorbid disorders $(N=13)$, somatoform disorder $(N=2)$, depression (chronic $N=1$, recurrent $N=10)$, dysthymic disorder $(N=1)$, specific phobias $(N=3)$, panic disorder $(N=3)$, anxiety disorder $(N=1)$, obsessive-compulsive disorder $(N=1)$, personality disorders (not otherwise specified $(N=2)$, mixed $(N=2)$, borderline personality disorder $(N=5)$, dependent and histrionic $(N=1))$, eating disorder $(N=3)$, sleeping disorder $(N=2)$, catalepsy $(N=1)$, psychogenic seizures $(N=1)$ and attention deficit disorder $(N=1)$. Of note, since approximately half of the DID participants did not have any other comorbidities apart from PTSD and other comorbidities were more randomly distributed across the sample, it is unlikely that these contribute to the classification in a systematic manner. Nevertheless, if this were to influence classification then our results are likely to represent an underestimation of classification strength and a more homogeneous sample would increase the sensitivity and specificity.

The DID and healthy control group were carefully matched for demographics including age, gender (all female), years of education and Western European ancestry. As previously shown, ${ }^{19}$ we did not find any significant differences between patients and healthy controls with respect to age or education (see Supplementary Table 1 available at https://doi.org/10.1192/bjp.2018.255). As part of the inclusion criteria, it was confirmed that all healthy controls were free of medication and psychiatric disorders. Furthermore, they scored below a critical cut-off of 25 on the Dissociative Experiences $\operatorname{Scale}^{26}$ and 29 on the Somatoform Dissociation Questionnaire. ${ }^{27}$ Depersonalisation symptoms were assessed using the Cambridge Depersonalisation Scale. ${ }^{28}$ Traumatic experiences were measured with the Traumatic Experience Checklist. ${ }^{29}$ As expected and previously reported, ${ }^{19}$ psychoform and somatoform dissociative symptoms and depersonalisation scores were significantly higher in the DID group as compared with healthy controls (all $P$-values $<0.001$ ). Individuals with DID also scored significantly higher compared with the controls for traumatic experiences (all
$P$-values $<0.001)$ on all five adverse life event categories, namely emotional neglect, emotional abuse, physical abuse, sexual abuse and sexual harassment (see Supplementary Table 1).

\section{MRI}

\section{Data acquisition}

All data were obtained on 3-T Philips whole-body MRI scanners (Philips Medical Systems, Best, Netherlands) in one of the three participating centres. An optimised structural MRI protocol ${ }^{30}$ with high reproducibility between centres was used at all three centres and T1-weighted anatomical MR scans were acquired (3D MPRAGE, repetition time $9.95 \mathrm{~ms}$, echo time $5.6 \mathrm{~ms}$, flip-angle $8^{\circ}$, $1 \times 1 \times 1 \mathrm{~mm}^{3}$ voxels, number of slices 160 , total scan time $10 \mathrm{~m} 14 \mathrm{~s}$ ).

DID patients and healthy controls were scanned interleaved (i.e. alternating between patients and healthy controls) within a short time interval. Final samples were distributed relatively equally over the three centres, with 10 patients and 17 healthy controls included from the UMCG, 7 patients and 11 healthy controls from the AMC and 15 patients and 15 healthy controls from Zurich. The number of participants in each group also did not differ across centres $\left(\chi^{2}=1.01, P=0.603\right)$.

\section{Image preprocessing}

After quality control checks, structural images were preprocessed using the SPM8 software (http://www.fil.ion.ucl.ac.uk/spm/, release 4667). They were first segmented into different tissue types via the 'new segment' tool, ${ }^{31}$ which is part of the voxel based morphology (VBM) processing pipeline. Rigidly aligned grey and white matter maps, down-sampled to $1.5 \mathrm{~mm}$ isotropic voxel size, were then used to diffeomorphically register all participants to their common average (i.e. study-specific template) by using a matching term that assumed a multinomial distribution. ${ }^{32}$ Registration involved estimating a set of initial velocities from which the deformations were computed by a geodesic shooting procedure. ${ }^{33}$ Classification was based on a set of 'scalar momentum' image features derived from this registration, which describe anatomical variability among participants. ${ }^{34}$ These images contain all information necessary to reconstruct the original images (in addition to the template) and therefore provide a parsimonious representation of shape difference. The scalar momentum images for grey and white matter were spatially smoothed with an isotropic $10 \mathrm{~mm}$ Gaussian kernel and concatenated prior to classification. This choice of feature construction method and smoothing level was based on previous work, where smoothed scalar momentum images yielded greater accuracy than a range of alternative features (including Jacobian determinants, rigidly aligned grey matter, spatially normalised grey matter and Jacobian scaled spatially normalised grey matter) for predicting participant age and gender in a publicly available data set ${ }^{35}$ and for discriminating neurological disorders from structural MRI. ${ }^{35}$

\section{Pattern recognition}

Binary Gaussian process classifiers $\left(\mathrm{GPCs}^{36}\right)$ were used as the primary analytical approach for this study. GPCs are a supervised pattern recognition approach that assigns a predictive probability of group membership to each individual based on a set of 'training' data. They are kernel classifiers similar to the widely used Support Vector Machines and have shown high levels of performance for neuroimaging data. ${ }^{37-39}$ Moreover, GPCs have advantages over Support Vector Machines in that the probabilistic predictions they provide encode a measure of predictive confidence that quantifies diagnostic uncertainty and can capture variability within clinical groups. More importantly, probabilistic predictions can be 
easily adjusted to compensate for the prior frequency of diagnostic classes in experimental populations. This means that inference remains coherent in classification scenarios where the frequency of each class in the test set is different from the frequencies observed in the training set and is useful to accommodate variations in disease prevalence ${ }^{40}$ and to compensate for unbalanced training data sets ${ }^{41}$ as outlined below. These properties are particularly important for the present study because the classes are unbalanced and the number of samples available for training is small.

Linear binary GPCs were used to discriminate people with DID from healthy controls. Technical details surrounding GPC inference have been presented elsewhere. A leave-one-participant-out crossvalidation approach was used to assess classifier generalisability, whereby a single participant was excluded to comprise the test set, and all parameters were inferred from the remaining data (training set), before applying this classifier to predict the labels for the test samples. Prior to classification, a $t$-test was used to select a set of discriminant voxels, using a fixed threshold of $P<0.001$. This was repeated, excluding each participant once. Importantly, feature selection was performed using the training data only, which ensures the classifier remains unbiased.

Classifier performance was evaluated using receiver operating characteristic curves derived from the probabilistic predictions and classification accuracy, which measures the classifier performance in a categorical sense. To derive categorical predictions from the probabilistic predictions obtained from the GPC, a threshold according to the frequency of classes in the training set was applied to the probabilistic predictions (i.e. 0.5 if the classes are balanced). Classifier assessment metrics included the sensitivity and specificity in addition to the positive and negative predictive value. Finally, the (balanced) accuracy was computed as the mean of sensitivity and specificity, which quantifies the overall categorical classification performance of the classifier in a way that accommodates potential class imbalance in the data.

\section{Forward maps of regional class differences}

To understand the relative importance of different brain regions underlying the classification decision, we employed a forward mapping strategy. ${ }^{42}$ This has advantages over the more commonly used strategy of directly mapping the weight vector. ${ }^{43}$ Specifically, a voxel may have a high weight because of an association with the class labels or as a result of high collinearity between voxels, where the high weight may help to cancel out noise or mismatch in other voxels. In contrast, the coefficients from the forward modelling approach proposed by Haufe and colleagues ${ }^{42}$ represent the group differences between classes, which are more often of interest when interpreting a trained classifier. In this case, stronger positive values in a forward map indicate a stronger association of a region with the DID ('favouring DID'), i.e. increased volume of a brain area in DID; whereas negative values indicate a stronger association with healthy controls ('favouring healthy controls'), i.e. decreased volume of a brain area in DID. Note that only voxels surviving the univariate feature selection step are included in the map and that the GPC discriminates data on the basis of the whole pattern. Hence, local inferences based on these approaches should be made with caution. To report results in standard Montreal Neurological Institute (MNI) coordinates, the DARTEL group tissue template (grey matter [GM], white matter [WM] and cerebrospinal fluid [CSF]) was coregistered to an SPM8 new_segment segmentation of the FSL MNI152_T1_1 mm template brain. Advanced normalisation tools (ANTS version 2.1.0 $0^{44}$ ) were used to calculate a non-linear transformation employing the SyN algorithm (antsRegistrationSyN.sh, default parameters) using multimodal information from the three channels (CSF, GM and WM). The results of the registration were visually inspected and it appeared to be a significant improvement on a simple affine registration. We selected the cluster peaks of the forward maps in terms of $x$-, $y$ - and $z$-coordinates on the basis of the maximum (absolute) coefficient. Anatomical labels were derived by A.A.T.S.R., S.C. and Y.R. S. from a consensus of different atlases.

\section{Results}

\section{Classification}

Using the imaging data, the classifier discriminated between the DID and healthy control groups with high sensitivity (71.88\%) and specificity (73.81\%), yielding an overall balanced accuracy of $72.84 \%$, which was significantly higher than the accuracy expected by chance $(P<0.01$, permutation test). Receiver operating characteristic analysis (see Fig. 1) further demonstrated that classification performance was above chance across all decision thresholds and yielded an area under the curve of 0.74 . The forward maps for the grey and white matter components are displayed in Fig. 2. Brain areas contributing to the classification are listed in Table 1.

\section{Relative decrease in regional volume in the DID group}

For grey matter, the pattern of voxels favouring healthy controls, i.e. a relative increase for the healthy control group or a relative decrease in the DID group, included bilateral middle, superior and dorsolateral frontal gyrus; left medial and right orbito-frontal gyrus; bilateral anterior cingulate gyrus; bilateral middle temporal gyrus; bilateral fusiform gyrus; right inferior temporal gyrus; left inferior parietal lobule and supramarginal gyrus and bilateral superior occipital gyrus.

For white matter, the pattern of voxels favouring healthy controls included the bilateral inferior fronto-occipital tract, the left corticospinal tract, and the right superior and left inferior longitudinal fasciculus. In addition, the white matter of the right inferior, bilateral middle and superior frontal regions; bilateral temporal, cerebellar and lateral occipital regions; the left amygdala-hippocampal junction and (anterior) cingulate were also included in the distinguishing pattern.

\section{Relative increase in regional volume for the DID group}

For grey matter, the pattern of voxels favouring DID was found to be far less pronounced than that of favouring healthy controls, and included left superior frontal gyrus, left medial parietal lobule and bilateral cerebellum.

The pattern of voxels in the white matter of the brain favouring DID included left inferior and superior longitudinal fasciculus, the left inferior fronto-occipital fasciculus and the right corticospinal tract. In addition, the white matter of bilateral (anterior) cingulate and insula regions, bilateral inferior, medial and superior frontal regions, left parietal regions and putamen, right inferior and middle temporal regions and bilateral cerebellum were included.

\section{Discussion}

\section{Main findings}

This is the first study to demonstrate that individuals with DID and healthy controls can be differentiated at the individual level with a high level of accuracy on the basis of their neuroanatomical markers. This discrimination was based on neuroanatomical data in the largest sample of individuals with DID included in a brain imaging study to date. We found widespread grey and white matter spatially dependent patterns of abnormal brain morphology in individuals with DID as compared with healthy controls. These 


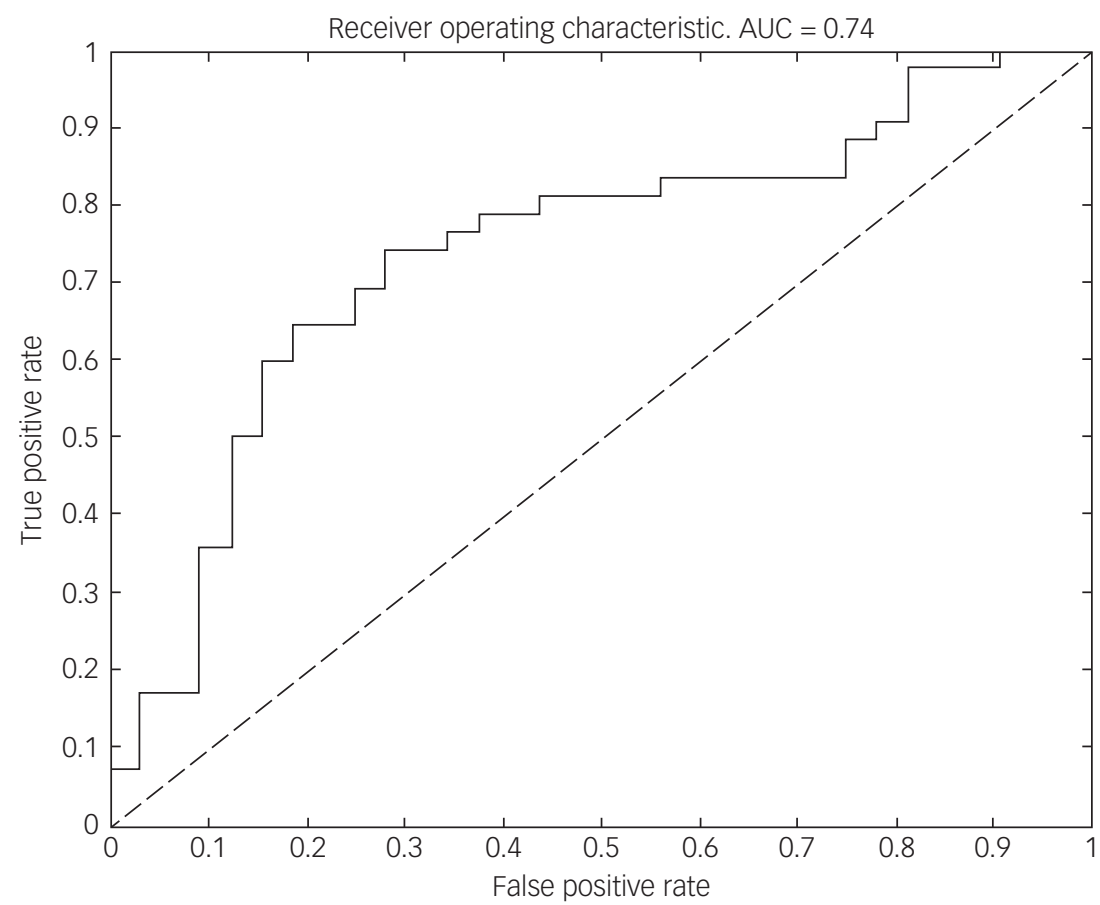

Fig. 1 Receiver operating characteristic curve for discriminating between people with dissociative identify disorder (DID) and healthy controls. The dotted line indicates chance level and the solid line is constructed by varying the decision threshold smoothly between 0 and 1 , plotting truepositive rate (sensitivity) against false-positive rate ( 1 - specificity). Points northwest of the dotted line indicate above chance discrimination. The area under the curve (AUC) summarises classifier performance across all decision thresholds.

findings are important because they provide evidence for a biological basis for distinguishing between genuine DID and healthy controls. The current study also provides support for the development of pattern recognition methodologies as a clinically useful diagnostic tool in DID, thereby paving the way for future studies with a diagnostic aim in distinguishing between genuine DID and other psychopathologies.

Applying pattern recognition methods to brain imaging data of people with DID offers a biomarker approach that can complement, aid and improve clinical decision making. This could reduce misdiagnoses, treatment time, treatment costs and ultimately improve the patient's quality of life.

\section{Comparison to other studies}

Despite the reported high prevalence of DID of approximately $6 \%$ in psychiatric out-patients ${ }^{45}$ and $1-3 \%$ of the general population, ${ }^{46}$ neurobiological studies on DID are scarce $e^{17,19,47}$ and depend on mass univariate data-analysis approaches, which provide inferences at the group level. Mass univariate data-analyses ignore information encoded by spatially distributed patterns of morphological abnormalities throughout the brain and have limited ability to provide inferences at the level of the individual. In contrast, multivariate data-analysis techniques using pattern classification methodology allow for the study of underlying patterns of effects and the distribution across brain regions and, more importantly, they provide predictions that quantify group separation at the individual level on the basis of patterns of abnormality in the data, which may be clinically useful. ${ }^{10}$

Pattern recognition methods are becoming widely used for neuroimaging-based psychiatric diagnostics. ${ }^{10}$ Our study shows that people with DID can be distinguished from healthy controls at an individual level with a sensitivity of $71.88 \%$ and specificity of $73.81 \%$. This level of accuracy is comparable to what has been demonstrated for most psychiatric disorders, ${ }^{10}$ including psychosis. $^{48}$

\section{Neuroanatomical biomarkers}

According to the Trauma Model, ${ }^{11}$ DID is an early-onset form of PTSD. ${ }^{11,17,18}$ Early life stressors may have long-lasting detrimental effects on neurobiology due to altered stress reactivity following childhood trauma. ${ }^{19}$ Earlier neuroanatomical studies in DID have found a smaller hippocampal volume, which seems to be the result of exposure to stress hormones due to antecedent traumatisation. Although it is currently unknown how early traumatisation affects the development of the brain, ${ }^{19}$ it is not surprising that long-lasting trauma results in widespread patterns of affected grey and white matter brain regions. We found affected brain regions in different lobules in the brain, but most prominently in the frontal grey and white matter regions, supporting findings from previous studies using univariate data-analysis approaches to investigate the neuroanatomical correlates of dissociative symptoms across disorders. ${ }^{17,49-51}$ Interestingly, the pattern of affected grey matter structural regions in the current study shows overlap with regions found in functional brain imaging studies involving dissociation, which included different patient samples and different paradigms - such as resting state in dissociative PTSD, ${ }^{52}$ script-driven imagery in $\mathrm{DID}^{14}$ or emotion processing in depersonalisation disorder $^{53}$ - but consistently reported functional aberrations in frontal regions of the brain. Models of trauma-related dissociation $^{14,52}$ propose important roles for the cingulate gyrus, medial prefrontal cortex and superior frontal regions in emotion under-/ overregulation and dissociation. The current multivariate study in the most severe form of dissociative disorders found that structural aberrations of these brain regions are part of the identified spatially distributed pattern. We therefore propose that future research into dissociation and DID focuses on these specific prefrontal areas, as 

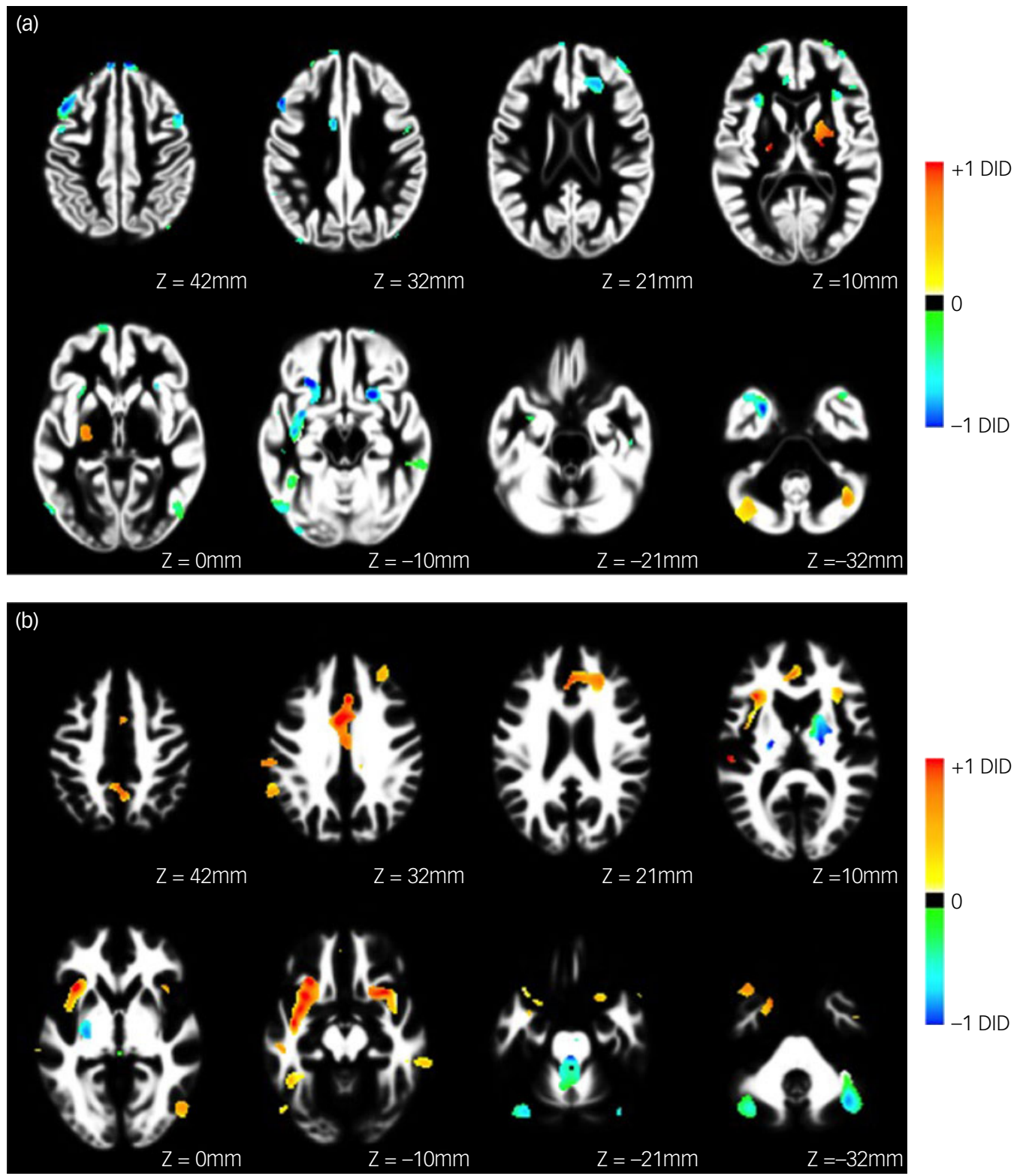

Fig. 2 Forward map showing the underlying pattern of abnormality differentiating people with dissociative identity disorder (DID) from normal healthy controls for (a) grey and (b) white matter. Coefficient images are overlaid on the study-specific anatomical templates in axial views. Images are scaled such that the maximum value in each image was equal to one, and only regions surviving the feature selection step are shown. Positive coefficients indicate a positive association in favour of DID, whereas negative coefficients indicate a positive association in favour of healthy controls.

well as the middle and dorsolateral prefrontal cortex, as important candidate regions for identifying neurobiomarkers of dissociation and specifically of DID.

\section{Limitations}

Our study has several strengths and limitations. First, although the findings show that a person with DID can be distinguished from healthy controls at an individual level with a high sensitivity and specificity, future studies are needed to unravel how individuals with DID differ from their most common misdiagnosed psychopathologies, such as borderline personality disorder and schizophrenia. We recommend that such future studies carefully debrief past psychiatric and neurological history, including depressive and/or psychotic episodes, history of epilepsy and duration of treatment, because these factors are likely to improve the ability for differential diagnostics of the classifier. The validation of the complex pattern recognition system with respect to these factors is important for clinical use and will need large patient samples of a wide variety of psychopathology. Furthermore, we recommend that future studies include a PTSD group without dissociative symptoms to investigate how PTSD comorbidity affects the classification, both at the level of the clinical presentation and biology. However, it is important to note that there is negligible overlap between results from the only multivariate study in $\mathrm{PTSD}^{54}$ and our results, indicating that the multivariate patterns found in the current study are likely to be dissociation-specific neuroanatomical biomarkers. Second, we employed a multicentre acquisition protocol to overcome single-site recruitment limitations, but carefully matched MRI acquisition parameters across sites using a study-optimised scanning sequence. ${ }^{30}$ We further accounted for inter-site effects during the data analyses by standardising the data within each site 


\begin{tabular}{|c|c|c|c|c|c|c|c|}
\hline & L/R & BA & $x$ & $y$ & $z$ & Volume & Max \\
\hline \multicolumn{8}{|l|}{$\mathrm{DID}<$ healthy controls ${ }^{\mathrm{a}}$} \\
\hline \multicolumn{8}{|l|}{ Grey matter } \\
\hline \multicolumn{8}{|l|}{ Frontal } \\
\hline I. frontal gyrus & L & 47 & -27.3 & 25.0 & -16.7 & 1889 & 20300 \\
\hline M. frontal gyrus & L & 10 & -25.0 & 63.5 & 10.5 & 50 & 14900 \\
\hline M. frontal gyrus & $\mathrm{R}$ & 10 & 37.4 & 58.2 & 16.4 & 324 & 16800 \\
\hline M. frontal gyrus & $\mathrm{R}$ & 8 & 45.0 & 14.7 & 52.7 & 366 & 19000 \\
\hline M. frontal gyrus/orbito-frontal gyrus & $\mathrm{R}$ & 11 & 23.0 & 16.5 & -14.5 & 319 & 18400 \\
\hline Medial frontal gyrus & L & 10 & -4.5 & 64.0 & -13.5 & 112 & 15200 \\
\hline Medial frontal gyrus & L & 9 & -4.5 & 56.0 & 40.5 & 98 & 17800 \\
\hline S./medial frontal gyrus & L & 10 & -6.0 & 65.5 & 22.5 & 31 & 15900 \\
\hline S. frontal gyrus & L & 6 & -6.7 & 1.5 & 73.3 & 40 & 14900 \\
\hline S. frontal sulcus & L & 6 & -23.0 & 17.3 & 66.3 & 18 & 13400 \\
\hline S. frontal sulcus & $\mathrm{R}$ & 6 & 26.5 & 5.5 & 70.0 & 188 & 16000 \\
\hline S. frontal gyrus & $\mathrm{R}$ & 9 & 8.0 & 53.0 & 45.0 & 172 & 19700 \\
\hline S. frontal gyrus & $\mathrm{R}$ & 8 & 7.5 & 42.0 & 56.0 & 25 & 11900 \\
\hline Dorsolateral prefrontal cortex/M. frontal gyrus & L & 9/46 & -51.0 & 23.5 & 33.5 & 814 & 18600 \\
\hline Dorsolateral prefrontal cortex/M. frontal gyrus & L & 9/46 & -40.5 & 41.0 & 2.5 & 75 & 15700 \\
\hline Dorsolateral prefrontal cortex/M. frontal gyrus & $\mathrm{R}$ & 9/46 & 44.5 & 30.3 & 15.7 & 324 & 16300 \\
\hline Cingulate gyrus & L & 24 & -9.0 & 3.0 & 35.0 & 70 & 17100 \\
\hline Anterior cingulate gyrus & L & 24 & -6.5 & 37.5 & 5.5 & 105 & 16400 \\
\hline Anterior cingulate gyrus & $\mathrm{R}$ & $24 / 32$ & 20.0 & 36.3 & 21.3 & 444 & 17100 \\
\hline Precentral gyrus & L & 4 & -55.0 & -9.5 & 51.5 & 126 & 16700 \\
\hline \multicolumn{8}{|l|}{ Parietal } \\
\hline I. parietal lobule & L & 40 & -50.5 & -54.0 & 54.0 & 25 & 15000 \\
\hline I. parietal lobule & L & 40 & -59.0 & -33.5 & 50.5 & 18 & 13900 \\
\hline Supramarginal gyrus & L & 40 & -63.0 & -47.3 & 33.3 & 16 & 17000 \\
\hline \multicolumn{8}{|l|}{ Occipital } \\
\hline I. occipital gyrus & L & 18 & -38.3 & -89.7 & -15.7 & 50 & 15400 \\
\hline M. occipital gyrus & L & 19 & -55.5 & -71.0 & -9.5 & 306 & 18500 \\
\hline S. occipital sulcus & L & 19 & -36.0 & -87.0 & 32.5 & 47 & 16300 \\
\hline S. occipital gyrus & $\mathrm{R}$ & 19 & 42.0 & -83.0 & 32.0 & 30 & 15700 \\
\hline \multicolumn{8}{|l|}{ Temporal } \\
\hline I. temporal gyrus & $\mathrm{R}$ & 20 & 45.3 & 1.5 & -43.8 & 99 & 18700 \\
\hline I. temporal gyrus/fusiform gyrus & $\mathrm{R}$ & 37 & 49.5 & -72.0 & -4.5 & 135 & 14200 \\
\hline I. temporal gyrus/fusiform gyrus & $\mathrm{R}$ & 20 & 49.8 & -18.4 & -23.4 & 10 & 14400 \\
\hline M. temporal gyrus & $\mathrm{R}$ & 21 & 61.3 & -35.0 & -5.3 & 259 & 17500 \\
\hline M./S. temporal gyrus & L & $21 / 38$ & -52.6 & 5.2 & -13.4 & 26 & 13200 \\
\hline M./S. temporal gyrus & $\mathrm{R}$ & $21 / 38$ & 38.0 & 13.5 & -41.5 & 140 & 16000 \\
\hline Fusiform gyrus & L & 37 & -40.0 & -46.5 & -6.5 & 171 & 15100 \\
\hline Other & & & & & & None & \\
\hline \multicolumn{8}{|l|}{ White matter } \\
\hline \multicolumn{8}{|l|}{ Tracts } \\
\hline I. Iongitudinal fasciculus & L & & -38.7 & -45.7 & -15.3 & 70 & 0.0669 \\
\hline S. Iongitudinal fasciculus (temporal part) & $\mathrm{R}$ & & 62.6 & -37.4 & -12.4 & 181 & 0.1720 \\
\hline Corticospinal tract & L & & -21.0 & -17.5 & 9.5 & 24 & 0.0807 \\
\hline I. frontal-occipital fasciculus & L & & -26.0 & 24.5 & 16.5 & 101 & 0.1190 \\
\hline I. frontal-occipital fasciculus & L & & -27.0 & 37.0 & 3.0 & 38 & 0.0686 \\
\hline I. frontal-occipital fasciculus & $\mathrm{R}$ & & 23.0 & 34.5 & 25.5 & 214 & 0.1120 \\
\hline \multicolumn{8}{|l|}{ Frontal } \\
\hline I. frontal white matter & $\mathrm{R}$ & & 23.0 & 14.5 & -19.5 & 179 & 0.1150 \\
\hline M. frontal white matter & $\mathrm{R}$ & & 58.0 & 32.3 & 18.3 & 73 & 0.0481 \\
\hline M. frontal white matter & L & & -53.0 & 17.0 & 41.5 & 488 & 0.1880 \\
\hline M. frontal white matter & $\mathrm{R}$ & & 49.5 & 12.0 & 52.5 & 183 & 0.1060 \\
\hline M./S. frontal white matter & $\mathrm{R}$ & & 44.7 & 48.7 & 25.5 & 61 & 0.0900 \\
\hline S. frontal white matter & L & & -11.5 & 70.5 & 0.0 & 76 & 0.1170 \\
\hline S. frontal white matter & L & & -4.5 & 47.0 & 52.5 & 56 & 0.1350 \\
\hline S. frontal white matter & L & & -24.0 & 33.0 & 56.0 & 37 & 0.1160 \\
\hline S. frontal white matter & L & & -6.0 & 68.3 & 22.3 & 31 & 0.1060 \\
\hline S. frontal white matter & L & & -23.0 & 17.3 & 66.3 & 18 & 0.0938 \\
\hline S. frontal white matter & L & & -25.5 & 65.5 & 7.5 & 12 & 0.0207 \\
\hline S. frontal white matter & $\mathrm{R}$ & & 24.3 & 1.0 & 72.3 & 111 & 0.2170 \\
\hline S. frontal white matter & $\mathrm{R}$ & & 9.0 & 50.0 & 50.0 & 103 & 0.1920 \\
\hline S. frontal white matter & $\mathrm{R}$ & & 23.4 & 68.8 & 10.4 & 27 & 0.0898 \\
\hline S. frontal white matter & $\mathrm{R}$ & & 9.3 & 38.0 & 59.7 & 25 & 0.2630 \\
\hline S. frontal/premotor white matter & $\mathrm{R}$ & & 8.0 & 17.0 & 70.0 & 54 & 0.1580 \\
\hline S. frontal/premotor white matter & L & & -6.5 & 1.5 & 76.0 & 40 & 0.1990 \\
\hline Anterior cingulate white matter & L & & -5.6 & 34.2 & 4.4 & 73 & 0.0713 \\
\hline Cingulate white matter & L & & -7.5 & -0.5 & 33.5 & 12 & 0.0112 \\
\hline Precentral white matter & L & & -47.6 & 1.6 & 52.6 & 85 & 0.0771 \\
\hline Insular white matter & $\mathrm{R}$ & & 29.0 & 21.5 & 13.5 & 36 & 0.0686 \\
\hline
\end{tabular}




\begin{tabular}{|c|c|c|c|c|c|c|c|}
\hline & L/R & BA & $x$ & $y$ & $z$ & Volume & Max \\
\hline \multicolumn{8}{|l|}{ Parietal } \\
\hline I. parietal lobule & L & & -57.0 & -33.5 & 53.5 & 13 & 0.0844 \\
\hline \multicolumn{8}{|l|}{ Occipital } \\
\hline Lateral occipital white matter & L & & -53.0 & -70.0 & -10.5 & 268 & 0.1300 \\
\hline Lateral occipital white matter (I. division) & L & & -40.5 & -89.5 & -11.5 & 50 & 0.1430 \\
\hline Lateral occipital white matter (S. division) & L & & -36.5 & -83.0 & 34.5 & 47 & 0.1130 \\
\hline Lateral occipital white matter (S. division) & $\mathrm{R}$ & & 40.3 & -75.3 & 46.0 & 30 & 0.1050 \\
\hline Lateral occipital white matter & $\mathrm{R}$ & & 52.0 & -74.0 & -11.0 & 124 & 0.1760 \\
\hline \multicolumn{8}{|l|}{ Temporal } \\
\hline I. temporal gyrus (anterior division) & $\mathrm{R}$ & & 47.0 & -8.5 & -45.0 & 40 & 0.0444 \\
\hline Temporal pole & L & & -42.0 & 17.5 & -34.0 & 98 & 0.0890 \\
\hline Temporal pole & L & & -54.0 & 13.0 & -19.0 & 21 & 0.1090 \\
\hline Temporal pole & $\mathrm{R}$ & & 31.5 & 13.3 & -41.7 & 140 & 0.2670 \\
\hline \multicolumn{8}{|l|}{ Other } \\
\hline Amygdala/hippocampus & L & & -26.5 & -7.0 & -20.5 & 1286 & 0.2750 \\
\hline Cerebellum (crus I) & L & & -32.8 & -71.6 & -33.0 & 61 & 0.0792 \\
\hline Cerebellum (crus I) & $\mathrm{R}$ & & 37.5 & -63.5 & -34.0 & 85 & 0.0857 \\
\hline \multicolumn{8}{|l|}{$\mathrm{DID}>$ healthy controls ${ }^{\mathrm{b}}$} \\
\hline \multirow{2}{*}{\multicolumn{8}{|c|}{ Grey matter }} \\
\hline \multicolumn{2}{|l|}{ Frontal } & & & & & & \\
\hline S. frontal sulcus & L & $4 / 6$ & -19.5 & -13.0 & 58.5 & 19 & 10600 \\
\hline \multicolumn{8}{|l|}{ Parietal } \\
\hline Medial posterior parietal & L & 7 & -14.7 & -58.0 & 57.7 & 16 & 14700 \\
\hline Medial posterior parietal/precuneus & L & 7 & -14.5 & -71.0 & 57.0 & 14 & 15200 \\
\hline Occipital & & & & & & None & \\
\hline Temporal & & & & & & None & \\
\hline \multicolumn{8}{|l|}{ Other } \\
\hline Cerebellum (crus I) & L & & -37.5 & -72.0 & -33.0 & 686 & 15500 \\
\hline Cerebellum (crus I) & $\mathrm{R}$ & & 42.5 & -63.5 & -37.0 & 285 & 16900 \\
\hline Periaqueductal grey & L & & -2.5 & -30.5 & -11.0 & 58 & 15000 \\
\hline White matter & & & & & & & \\
\hline Tracts & & & & & & & \\
\hline I. Iongitudinal fasciculus & L & & -41.5 & -47.0 & -4.5 & 98 & 0.1060 \\
\hline I. Iongitudinal fasciculus & L & & -42.5 & -5.0 & -20.5 & 14 & 0.0369 \\
\hline S. Iongitudinal fasciculus & L & & -50.5 & -52.5 & 54.0 & 22 & 0.0608 \\
\hline S. Iongitudinal fasciculus & L & & -19.5 & -14.4 & 61.0 & 19 & 0.3820 \\
\hline Corticospinal tract & $\mathrm{R}$ & & 16.3 & -2.7 & 9.5 & 438 & 0.2030 \\
\hline I. fronto-occipital fasciculus & L & & -39.9 & 40.6 & 0.6 & 37 & 0.1390 \\
\hline Frontal & & & & & & & \\
\hline I. frontal white matter & L & & -32.5 & 23.7 & -19.7 & 58 & 0.0835 \\
\hline I. frontal white matter & L & & -21.7 & 12.0 & -18.3 & 37 & 0.0399 \\
\hline I. frontal white matter (pars triangularis) & $\mathrm{R}$ & & 56.7 & 28.7 & 13.5 & 250 & 0.1070 \\
\hline M. frontal white matter & $\mathrm{R}$ & & 44.0 & 17.7 & 51.7 & 174 & 0.1520 \\
\hline Medial frontal white matter & L & & -4.6 & 54.6 & 40.6 & 42 & 0.0950 \\
\hline Medial frontal/forceps minor & L & & -7.8 & 62.6 & -11.6 & 35 & 0.0564 \\
\hline Medial frontal/forceps minor & $\mathrm{R}$ & & 6.5 & 54.7 & 37.7 & 65 & 0.1820 \\
\hline S. frontal white matter & L & & -33.7 & 29.0 & 48.7 & 274 & 0.1390 \\
\hline S. frontal white matter & L & & -25.5 & 64.0 & 14.5 & 37 & 0.0903 \\
\hline S. frontal white matter & $\mathrm{R}$ & & 36.0 & 55.7 & 19.7 & 233 & 0.1720 \\
\hline S. frontal/premotor white matter & $\mathrm{R}$ & & 22.3 & 9.5 & 65.5 & 18 & 0.0396 \\
\hline Precentral/premotor white matter & L & & -46.5 & 2.5 & 46.5 & 27 & 0.1040 \\
\hline Precentral/premotor white matter & L & & -56.4 & -7.8 & 49.4 & 14 & 0.0527 \\
\hline Cingulate white matter & L & & -11.0 & 8.0 & 36.5 & 57 & 0.1240 \\
\hline Anterior cingulate white matter & L & & -7.8 & 38.4 & 0.6 & 31 & 0.0572 \\
\hline Cingulate white matter/forceps minor & $\mathrm{R}$ & & 15.4 & 31.4 & 25.8 & 207 & 0.1120 \\
\hline Insula white matter & L & & -31.5 & 23.5 & 11.0 & 172 & 0.1480 \\
\hline Insula white matter & $\mathrm{R}$ & & 33.5 & 29.5 & 9.0 & 97 & 0.1210 \\
\hline Parietal & & & & & & & \\
\hline S. parietal lobule & L & & -15.0 & -55.5 & 53.5 & 16 & 0.2130 \\
\hline Supramarginal white matter (posterior division) & L & & -63.0 & -47.3 & 33.3 & 16 & 0.0923 \\
\hline Precuneus white matter & L & & -14.0 & -69.5 & 53.5 & 14 & 0.1860 \\
\hline Occipital & & & & & & & \\
\hline M. occipital white matter & $\mathrm{R}$ & & 45.4 & -70.4 & -4.3 & 9 & 0.0510 \\
\hline Temporal & & & & & & & \\
\hline Temporal pole & L & & -33.4 & 14.4 & -38.6 & 98 & 0.0626 \\
\hline I. Temporal white matter (anterior division) & $\mathrm{R}$ & & 46.6 & 1.4 & -45.4 & 56 & 0.0755 \\
\hline M. Temporal white matter & $\mathrm{R}$ & & 49.0 & -34.0 & -8.0 & 77 & 0.1490 \\
\hline Fusiform white matter & L & & -49.2 & -66.6 & -17.4 & 36 & 0.0401 \\
\hline Other & & & & & & & \\
\hline Cerebellum (crus I) & L & & -45.5 & -55.0 & -28.0 & 624 & 0.3260 \\
\hline Cerebellum (crus I) & $\mathrm{R}$ & & 44.5 & -65.3 & -41.3 & 195 & 0.1170 \\
\hline
\end{tabular}


Table 1 (Continued)

\begin{tabular}{|c|c|c|c|c|c|c|c|}
\hline & L/R & BA & $x$ & $y$ & $z$ & Volume & Max \\
\hline Putamen & L & & -31.0 & -12.0 & 1.0 & 312 & 0.2000 \\
\hline Brainstem (midbrain) & L & & -7.0 & -29.0 & -9.5 & 58 & 0.1650 \\
\hline
\end{tabular}

separately prior to classification, which speaks against the possibility that inter-site effects can be fully attributed to site effects. Third, only women volunteered to participate in the study and therefore the results cannot be extended to all people with DID. Most of these women were currently using medication or had used medication in the past (see Supplementary Table 1). However, because medication use was highly variable across participants it is unlikely that the effects on grey and white matter will be consistent, as previously reported in a separate study of our group. ${ }^{17}$ Nevertheless, it is recommended that future studies systematically investigate whether neuroanatomical changes are related to psychotropic medication.

Antje A. T. S. Reinders, PhD (iD), Senior Research Associate with Lecturer status, Department of Psychological Medicine, Institute of Psychiatry, Psychology and Neuroscience, King's College London, UK and Department of Neuroscience, University Medical Center Groningen, University of Groningen, The Netherlands; Andre F. Marquand, PhD, Assistant Professor, Donders Institute for Brain Cognition and Behaviour, Radboud University, The Netherlands and Honorary Lecturer, Department of Clinical Neuroscience, Centre for Neuroimaging Sciences, Institute of Psychiatry, Psychology and Neuroscience, King's College London, UK; Yolanda R. Schlumpf, PhD, Postdoctoral Assistant, Division of Neuropsychology, Department of Psychology, University of Zurich and Clienia Litenheid AG, Private Clinic for Psychiatry and Psychotherapy, Switzerland; Sima Chalavi, PhD, Postdoctoral Researcher, Department of Neuroscience, University Medical Center Groningen, University of Groningen, The Netherlands and Research Center for Movement Control and Neuroplasticity, Department of Movement Sciences, Katholieke Universiteit Leuven, Belgium; Eline M. Vissia, PhD, Mental Healthcare Psychologist, Department of Neuroscience, University Medical Center Groningen, University of Groningen and Top Referent Trauma Centrum, GGz Centraal, The Netherlands; Ellert R. S. Nijenhuis, PhD, Psychologist/ Psychotherapist, Clienia Littenheid AG, Private Clinic for Psychiatry and Psychotherapy، Switzerland; Paola Dazzan, PhD, Professor of Neurobiology of Psychosis, Vice Dean International, Honorary Consultant Psychiatrist, Department of Psychosis Studies, Institute of Psychiatry, King's College London, UK; Lutz Jäncke, PhD, Professor of Neuropsychology, Scientific Director, Clienia Littenheid AG, Private Clinic for Psychiatry and Psychotherapy and Research Unit for Plasticity and Learning of the Healthy Aging Brain, University of Zurich, Switzerland; Dick J. Veltman, PhD, Professor of Neuroimaging in Psychiatry, Department of Psychiatry, VU University Medical Center, The Netherlands.

Correspondence: Antje A. T. S. Reinders, Department of Psychological Medicine, Institute of Psychiatry, Psychology and Neuroscience, King's College London, De Crespigny Park, London SE5 8AZ, UK. Email: a.a.t.s.reinders@kcl.ac.uk

First received 16 Jan 2018, final revision 24 Sep 2018, accepted 14 Oct 2018

\section{Supplementary material}

Supplementary material is available online at https://doi.org/10.1192/bjp.2018.255.

\section{Funding}

This article represents independent research part funded by the National Institute for Health Research Biomedical Research Centre at South London and Maudsley National Health Service Foundation Trust and King's College London. The views expressed are those of the author(s) and not necessarily those of the National Health Service, the National Institute for Health Research or the Department of Health. A.A.T.S.R. was supported by the Netherlands Organisation for Scientific Research (www.nwo.nl), NWO-VENI grant number 451-07-009. S.C. is supported by a David Caul graduate research grant from the International Society for the Study of Trauma and Dissociation (http://www.isst-d.org/about/awards.htm). A.F.M. gratefully acknowledges support from the King's College London Centre of Excellence in Medical Engineering, funded by the Wellcome Trust and Engineering and Physical Sciences Research Council and from a VIDI fellowship from the Netherlands Organisation for Scientific Research (grant number 016.156.415). Y.R.S. was supported by the Forschungskredit of the University of Zurich.

\section{Acknowledgements}

The authors thank all the participants and their therapists. We thank Nel Draijer, Mechteld Giesen, Ekaterina Weder and Eva Zimmermann for participant inclusion and scanning, as well as Andrew Lawrence for coordinate transformation into MNI space and for preparing the Tables.

\section{References}

1 Gillig PM. Dissociative identity disorder: a controversial diagnosis. Psychiatry 2009; 6: 24-9.

2 Dalenberg CJ, Brand BL, Loewenstein RJ, Gleaves DH, Dorahy MJ, Cardeña E, et al. Reality versus fantasy: reply to Lynn et al. (2014). Psychol Bull 2014; 140: 911-20.

3 Brand BL, Vissia EM, Chalavi S, Nijenhuis ERS, Webermann AR, Draijer N, et al. DID is trauma based: further evidence supporting the trauma model of DID. Acta Psychiatr Scand 2016; 134: 560-3.

4 International Society for the Study of Trauma and Dissociation. Guidelines for treating dissociative identity disorder in adults, third revision. J Trauma Dissociation 2011; 12: 115-87.

5 Piper A, Merskey $\mathrm{H}$. The persistence of folly: a critical examination of dissociative identity disorder. Part I. The excesses of an improbable concept. Can J Psychiatry 2004; 49: 592-600.

6 Pope HG, Barry S, Bodkin A, Hudson Jl. Tracking scientific interest in the dissociative disorders: a study of scientific publication output 1984-2003. Psychother Psychosom 2006; 75: 19-24.

7 Sar V. Epidemiology of dissociative disorders: an overview. Epidemiol Res Int 2011; 1: 1-8.

8 Lloyd M. Reducing the cost of dissociative identity disorder: measuring the effectiveness of specialized treatment by frequency of contacts with mental health services. J Trauma Dissociation 2016; 17: 362-70.

9 Myrick AC, Webermann AR, Langeland W, Putnam FW, Brand BL. Treatment of dissociative disorders and reported changes in inpatient and outpatient cost estimates. Eur J Psychotraumatol 2017; 8: 1375829.

10 Wolfers T, Buitelaar JK, Beckmann C, Franke B, Marquand AF. From estimating activation locality to predicting disorder: a review of pattern recognition for neuroimaging-based psychiatric diagnostics. Neurosci Biobehav Rev 2015; 57 : 328-49.

11 Vissia EM, Giesen ME, Chalavi S, Nijenhuis ERS, Draijer N, Brand BL, et al. Is it Trauma- or Fantasy-based? Comparing dissociative identity disorder, posttraumatic stress disorder, simulators, and controls. Acta Psychiatr Scand 2016; 134: 111-28.

12 Schlumpf YR, Nijenhuis ERS, Chalavi S, Weder EV, Zimmermann E, Luechinger $\mathrm{R}$, et al. Dissociative part-dependent biopsychosocial reactions to backward masked angry and neutral faces: an fMRI study of dissociative identity disorder. Neurolmage Clin 2013; 3: 54-64.

13 Schlumpf YR, Reinders AATS, Nijenhuis ERS, Luechinger R, van Osch MJP, Jäncke $L$. Dissociative part-dependent resting-state activity in dissociative identity disorder: a controlled FMRI perfusion study. PLOS One 2014; 9: e98795.

14 Reinders AATS, Willemsen ATM, Vissia EM, Vos HPJ, den Boer JA, Nijenhuis ERS. The psychobiology of authentic and simulated dissociative personality states: the full Monty. J Nerv Ment Dis 2016; 204: 445-57.

15 Zotev V, Phillips R, Young KD, Drevets WC, Bodurka J. Prefrontal control of the amygdala during real-time fMRI neurofeedback training of emotion regulation. PLOS One 2013; 8: e79184.

16 Kim S, Birbaumer N. Real-time functional MRI neurofeedback: a tool for psychiatry. Curr Opin Psychiatry 2014; 27: 332-6.

17 Chalavi S, Vissia EM, Giesen ME, Nijenhuis ERS, Draijer N, Barker GJ, et al. Similar cortical but not subcortical gray matter abnormalities in women with posttraumatic stress disorder with versus without dissociative identity disorder. Psychiatry Res 2015; 231: 308-19. 
18 Chalavi S, Vissia EM, Giesen ME, Nijenhuis ERS, Draijer N, Cole JH, et al. Abnormal hippocampal morphology in dissociative identity disorder and posttraumatic stress disorder correlates with childhood trauma and dissociative symptoms. Hum Brain Mapp 2015; 36: 1692-704.

19 Reinders AATS, Chalavi S, Schlumpf YR, Vissia EM, Nijenhuis ERS, Jäncke L, et al. Neurodevelopmental origins of abnormal cortical morphology in dissociative identity disorder. Acta Psychiatr Scand 2018; 137: 157-70.

20 American Psychiatric Association. Diagnostic and Statistical Manual of Mental Disorders, DSM-5 (5th edn). American Psychiatric Association, 2013.

21 Boon S, Draijer N. Multiple personality disorder in The Netherlands: a clinical investigation of 71 patients. Am J Psychiatry 1993; 150: 489-94.

22 Steinberg M. Structured Clinical Interview for DSM-IV Dissociative Disorders (SCID-D). American Psychiatric Press, 1993.

23 Draijer N, Boon S. The imitation of dissociative identity disorder: patients at risk, therapists at risk. J Psychiatry Law 1999; 27: 423-58.

24 Rodewald F, Wilhelm-Göling C, Emrich HM, Reddemann L, Gast U. Axis-I comorbidity in female patients with dissociative identity disorder and dissociative identity disorder not otherwise specified. J NerV Ment Dis 2011; 199 122-31.

25 Bozkurt H, Duzman Mutluer T, Kose C, Zoroglu S. High psychiatric comorbidity in adolescents with dissociative disorders. Psychiatry Clin Neurosci 2015; 69 . 369-74.

26 Bernstein EM, Putnam FW. Development, reliability, and validity of a dissociation scale. J Nerv Ment Dis 1986; 174: 727-35.

27 Nijenhuis ERS, Spinhoven $P$, Van Dyck R, Van der Hart O, Vanderlinden J. The development and psychometric characteristics of the Somatoform Dissociation Questionnaire (SDQ-20). J Nerv Ment Dis 1996; 184: 688-94.

28 Sierra M, Berrios GE. The Cambridge Depersonalization Scale: a new instrument for the measurement of depersonalization. Psychiatry Res 2000; 93 : 153-64.

29 Nijenhuis ERS, Van der Hart O, Kruger K. The psychometric characteristics of the Traumatic Experiences Checklist (TEC): first findings among psychiatric outpatients. Clin Psychol Psychother 2002; 9: 200-10.

30 Chalavi S, Simmons A, Dijkstra H, Barker GJ, Reinders AA. Quantitative and qualitative assessment of structural magnetic resonance imaging data in a two-center study. BMC Med Imaging 2012; 12: 27

31 Ashburner J, Friston KJ. Unified segmentation. Neuroimage 2005; 26: 839-51.

32 Ashburner J, Friston KJ. Computing average shaped tissue probability templates. Neuroimage 2009; 45: 333-41.

33 Ashburner J, Friston KJ. Diffeomorphic registration using geodesic shooting and Gauss-Newton optimisation. Neuroimage 2011; 55: 954-67.

34 Singh N, Fletcher PT, Preston JS, Ha L, King R, Marron JS, et al. Multivariate Statistical Analysis of Deformation Momenta Relating Anatomical Shape to Neuropsychological Measures. In 13th International Conference on Medical Image Computing and Computer-Assisted Intervention, MICCAI, 2010: 529-37.

35 Marquand AF, Filippone M, Ashburner J, Girolami M, Mourao-Miranda J, Barker GJ, et al. Automated, high accuracy classification of parkinsonian disorders: a pattern recognition approach. PLoS One 2013; 8: e69237.

36 Rasmussen CE, Williams CKI. Gaussian Processes for Machine Learning. University Press Group Limited, 2006.

37 Marquand A, Howard M, Brammer M, Chu C, Coen S, Mourão-Miranda J. Quantitative prediction of subjective pain intensity from whole-brain fMRI data using Gaussian processes. Neuroimage 2010; 49: 2178-89.
38 Orrù G, Pettersson-Yeo W, Marquand AF, Sartori G, Mechelli A. Using Support Vector Machine to identify imaging biomarkers of neurological and psychiatric disease: a critical review. Neurosci Biobehav Rev 2012; 36: 1140-52.

39 Schrouff J, Rosa MJ, Rondina JM, Marquand AF, Chu C, Ashburner J, et al. PRONTO: pattern recognition for neuroimaging toolbox. Neuroinformatics 2013; 11: 319 .

40 Hahn T, Marquand AF, Plichta MM, Ehlis A-C, Schecklmann MW, Dresler T, et al. A novel approach to probabilistic biomarker-based classification using functional near-infrared spectroscopy. Hum Brain Mapp 2013; 34: 1102.

41 Lim HK, Jung WS, Ahn KJ, Won WY, Hahn C, Lee SY, et al. Regional cortical thickness and subcortical volume changes are associated with cognitive impairments in the drug-naive patients with late-onset depression. Neuropsychopharmacology 2012; 37: 838-49.

42 Haufe S, Meinecke F, Gorgen K, Dahne S, Haynes J-D, Blankertz B, et al. On the interpretation of weight vectors of linear models in multivariate neuroimaging. Neuroimage 2014; 87: 96-110.

43 Mourão-Miranda J, Bokde ALW, Born C, Hampel H, Stetter M. Classifying brain states and determining the discriminating activation patterns: support Vector Machine on functional MRI data. Neuroimage 2005; 28: 980-95.

44 Avants BB, Tustison NJ, Song G, Cook PA, Klein A, Gee JC. A reproducible evaluation of ANTs similarity metric performance in brain image registration. Neuroimage 2011; 54: 2033-44.

45 Foote B, Smolin Y, Kaplan M, Legatt ME, Lipschitz D. Prevalence of dissociative disorders in psychiatric outpatients. Am J Psychiatry 2006; 163: 623-9.

46 Sar V, Akyüz G, Doğan O. Prevalence of dissociative disorders among women in the general population. Psychiatry Res 2007; 149: 169-76.

47 Dorahy MJ, Brand BL, Sar V, Krüger C, Stavropoulos P, Martínez-Taboas A, et al. Dissociative identity disorder: an empirical overview. Aust N Z J Psychiatry 2014; 48: 402-17.

48 Mourao-Miranda J, Reinders AATS, Rocha-Rego V, Lappin J, Rondina J, Morgan C, et al. Individualized prediction of illness course at the first psychotic episode: a support vector machine MRI study. Psychol Med 2012; 42: 1037-47.

49 Nardo D, Högberg G, Lanius RA, Jacobsson H, Jonsson C, Hällström T, et al. Gray matter volume alterations related to trait dissociation in PTSD and traumatized controls. Acta Psychiatr Scand 2013; 128: 222-33.

50 Sierra M, Nestler S, Jay E-L, Ecker C, Feng Y, David AS. A structural MRI study of cortical thickness in depersonalisation disorder. Psychiatry Res Neuroimaging 2014; 224: 1-7.

51 Daniels JK, Frewen P, Theberge J, Lanius RA. Structural brain aberrations associated with the dissociative subtype of post-traumatic stress disorder. Acta Psychiatr Scand 2016; 133: 232-40.

52 Lanius RA, Vermetten E, Loewenstein RJ, Brand B, Schmahl C, Bremner JD, et al. Emotion modulation in PTSD: clinical and neurobiological evidence for a dissociative subtype. Am J Psychiatry 2010; 167: 640-7.

53 Lemche E, Anilkumar A, Giampietro VP, Brammer MJ, Surguladze SA, Lawrence NS, et al. Cerebral and autonomic responses to emotional facial expressions in depersonalisation disorder. Br J Psychiatry 2008; 193: 222-8.

54 Gong Q, Li L, Tognin S, Wu Q, Pettersson-Yeo W, Lui S, et al. Using structura neuroanatomy to identify trauma survivors with and without post-traumatic stress disorder at the individual level. Psychol Med 2014; 44: 195-203. 